\title{
A First-principles Study on Magnetism of Al Impurity in bec Fe
}

\author{
Gul Rahman ${ }^{1}$ and In Gee Kim ${ }^{2 *}$ \\ ${ }^{1}$ Department of Physics, Quaid-i-Azam University, Islamabad, Pakistan \\ ${ }^{2}$ Graduate Institute of Ferrous Technology, Pohang University of Science and Technology, Pohang 790-784, Korea
}

(Received 8 November 2010, Received in final form 10 February 2011, Accepted 10 February 2011)

\begin{abstract}
The magnetism and electronic structure of bec $\mathrm{Al}_{1} \mathrm{Fe}_{26}$ was investigated by means of first-principles calculations with and without spin-orbit coupling (SOC). From the calculated total energy, the SOC corrected system is shown to be approximately $5 \mathrm{meV}$ per atom lower than the SOC uncorrected system. The induced spin magnetic moment at the Al site was $-0.125 \mu_{B}$ without SOC and $-0.124 \mu_{B}$ with SOC. The orbital magnetic moments were calculated to be $0.002 \mu_{\mathrm{B}}$ in $[\overline{100}]$ direction for $\mathrm{Al}$. The electronic structures showed the nearest neighbor antiferromagnetic interaction between $\mathrm{Fe}$ and $\mathrm{Al}$ to be essential for determining the magnetism of the $\mathrm{Al}_{1} \mathrm{Fe}_{26}$ system.
\end{abstract}

Keywords: Fe, Al impurity, magnetism, spin-orbit coupling, first-principles

\section{Introduction}

An iron-aluminum system in its Fe-rich portion is interesting given its complex magnetic phase diagram and order-disorder transformation. Alloys based on the Fe-Al intermetallic phases matrix are becoming increasingly popular in terms of practical usage due to their high resistance to oxidation, carbonization, and sulfurization processes at high temperatures and their resistance to the effects of exposition to liquid salts [1]. Another important factor deciding application is the low cost of their main components, iron and aluminum. A high content of aluminum also lowers the intrinsic alloy density, which correspondingly lowers the weight of the components constituting the alloy [1-3].

However, high Al contents can create many side effects on steel (complicated Fe-Al compounds, Fe-Al-C compounds, i.e., $\kappa$-carbide), which greatly affect the creep properties or deformation mechanics. These technological problems have thus been discussed in the literature. It is also well known that $\mathrm{Al}$ is a ferrite former through destabilization of the austenite domain. Contrary to the $3 d$ alloying elements in bcc Fe [4], the group III-VI elements show strong spin-orbit coupling (SOC) effects, especially upon magnetism, Si in bcc Fe [5]. Newly developed lightweight

*Corresponding author: Tel: +82-54-279-9014

Fax: +82-54-279-9099, e-mail: igkim@postech.ac.kr steels based on ferritic iron aluminum alloys show promising physical, mechanical, and technological properties such as high specific elastic stiffness and strength, excellent ductility and formability, reduced specific weight, and an improved corrosion resistance. However, the environmental embrittlement at room temperature regions [6] has not been solved yet.

Instead of addressing the room temperature ductility of $\mathrm{Fe}-\mathrm{Al}$ intermetallic phases, new functional properties of the Fe-Al system, its relatively high magnetostriction near 20 at $\%$ aluminum contents, has been considered [7, 8]. The observed high magnetostriction is considered to be responsible for the transition from a disordered to an ordered phase by increasing aluminum contents. However, the effects of the intrinsic magnetic properties on the dilute alloying region are seldom considered. As such, it is essential to study the effects of $\mathrm{Al}$ on the electronic structure and magnetism of bcc Fe, important not only from an applications point of view, but also from a fundamental understanding point of view. Here, we not only investigate the electronic and magnetic properties of bcc Fe-Al, but also the effects of SOC on bcc Fe-Al magnetism.

\section{Computational Method}

A $3 \times 3 \times 3$ supercell of bcc $\mathrm{Fe}$, corresponding to $27 \mathrm{Fe}$ atoms within the unit cell, was considered for $\mathrm{Al}$ sub- 


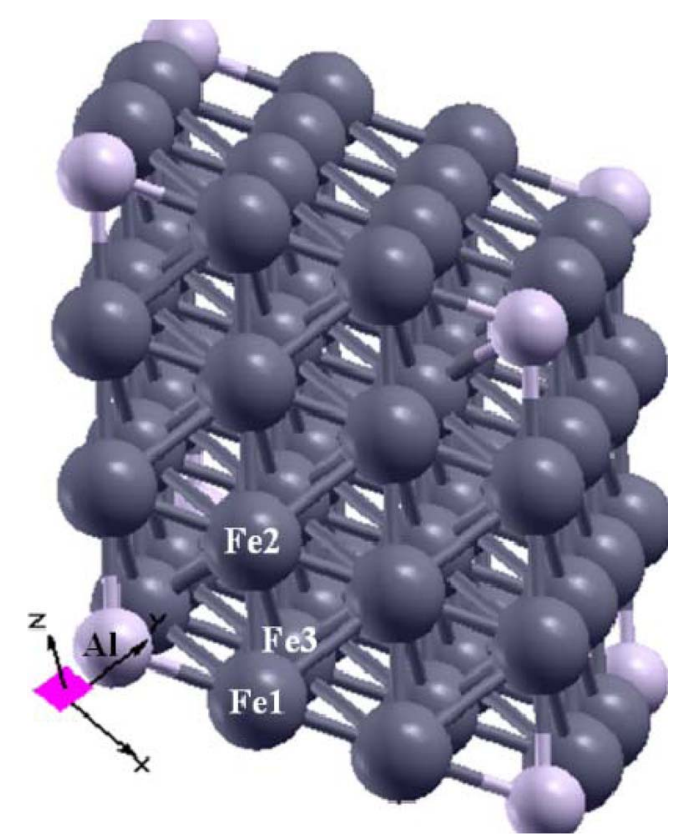

Fig. 1. The body centered cubic (bcc) $3 \times 3 \times 3$ supercell model. The Fe atoms are represented by dark spheres, while the $\mathrm{Al}$ impurity is represented by light spheres.

stitution of the body-centered $\mathrm{Fe}$ atom of the supercell; henceforth, we denote the model as $\mathrm{Al}_{1} \mathrm{Fe}_{26}$. This $\mathrm{Al}$ concentration corresponded to $3.7 \mathrm{wt} \%$ of $\mathrm{Al}$ in bcc Fe. The supercell is shown in Fig. 1. In such a cubic unit cell, whose space group becomes $\operatorname{Im} \overline{3} m, \# 229$ by Al substitution at the central Fe atom, the (2a) Wyckoff position, it has the eight nearest-neighbor $\mathrm{Fe}$ atoms on the $(16 f)$ Wyckoff sites, the six second-nearest-neighbors on the (12e) Wyckoff sites, and the 12 third-nearest-neighbors on (24h) Wyckoff sites. These Fe atoms are denoted as Fe1, $\mathrm{Fe} 2$, and $\mathrm{Fe} 3$, and are the 1st, 2nd, and 3rd nearest neighbors, respectively.

The Kohn-Sham equations were solved in the framework of the full potential linearized augmented plane wave (FLAPW) $[9,10]$ method under the generalized gradient approximation (GGA) [11]. An energy cutoff of $4.0(2 \pi / a)$, where $a$ is the lattice parameter of each calculation, was employed for expanding the linearized augmented plane wave (LAPW) basis set. This plane wave cutoff corresponds to 2350 LAPWs per k-point and spin. A $16.1245(2 \pi / a)$ cutoff was used for the star functions depicting the charge density and potential in the interstitial regions. Lattice harmonics with $l \leq 8$ were employed to expand the charge density, potential, and wave functions inside each muffin-tin (MT) sphere, with a radius of 2.2 a.u. for all the atoms. Integrations inside the Brillouin zone (BZ) were performed using the improved tetrahedron method [12] over a $13 \times 13 \times 13$ mesh within the three-dimensional 3D-BZ, corresponding to 84 k-points inside the irreducible wedge of the 3D-BZ.

All core electrons were first treated fully relativistically and valence states scalar relativistically, without spin-orbit coupling (SOC) [13]. For spin-orbit coupling on valence states, we then employed the second variation method [14] with the spin diagonal parts of the density subjected to a self-consistency loop. During the second variation procedure, integrations inside the 3D-BZ were executed in the full-BZ, the 1099 k-points. The explicit orthogonalization (XO) scheme was employed to ensure the orthogonality between the core and valence states [15].

All atoms were fully relaxed at each lattice volume until the atomic forces on each atom were less than 2 mRy/a.u. The selected computational parameters fulfilled the convergence criterion [16]. The equilibrium lattice constants and bulk moduli $B$ were determined by the fifteen-point fitting of the total energy and volume to the Birch-Murnaghan equation of states [17]. Using the optimized lattice constants, further calculations were carried out with and without SOC.

\section{Results and Discussions}

Calculations were carried out in the nonmagnetic (NM) and ferromagnetic (FM) states, with the total energy calculations indicating the FM state to be more stable than the NM state at the optimized lattice constant. Considering the effects of the SOC, the authors observed that the FM state with SOC to be approximately $5 \mathrm{meV}$ per atom lower than the FM state without SOC. Accordingly, we will focus only on the FM state with and without SOC, and the attention will not be given to the NM and FM states without structural relaxation as they were higher in energy.

The lattice parameters of the bcc $\mathrm{Al}_{1} \mathrm{Fe}_{26}$ were optimized and the calculated lattice parameter was $8.513 \AA$ without SOC or atomic relaxation. However, this value changed to $8.510 \AA$ when the atomic relaxation was allowed, indicating that the atomic relaxation slightly changed the volume of the unit cell. The approximately same value $(8.510 \AA)$ was obtained upon repeating the calculations with SOC and atomic relaxation. Therefore, SOC does not bear any significant effect upon the $\mathrm{Al}_{1} \mathrm{Fe}_{26}$ lattice constant. From the equation of state [17], we also estimated the bulk modulus $B$ to be $175.41 \mathrm{GPa}$ without SOC, and when the SOC was allowed, $B$ was calculated to be $175.95 \mathrm{GPa}$. This shows that the solid solution hardening is not as significant at this $\mathrm{Al}$ content compared to that (175.75 GPa) of the pure bcc Fe [4].

The calculated magnetic moments within the MT sphere 
Table 1. Calculated spin magnetic moments in units of $\mu_{B}$ inside each muffin-tin (MT) sphere of $\mathrm{Al}_{1} \mathrm{Fe}_{26}$ with spin-orbit coupling (SOC) and without SOC cases.

\begin{tabular}{ccccc}
\hline \hline Case & $\mathrm{Al}$ & $\mathrm{Fe} 1$ & $\mathrm{Fe} 2$ & $\mathrm{Fe} 3$ \\
\hline With SOC & -0.124 & 2.110 & 2.224 & 2.223 \\
Without SOC & -0.125 & 2.112 & 2.227 & 2.226 \\
\hline
\end{tabular}

Table 2. Calculated orbital magnetic moments with SOC in units of $\mu_{\mathrm{B}}$ inside each muffin-tin (MT) sphere of: (a) unrelaxed; (b) relaxed $\mathrm{Al}_{1} \mathrm{Fe}_{26}$.

(a)

\begin{tabular}{cccc}
\hline \hline Atom & $L_{x}$ & $L_{y}$ & $L_{z}$ \\
\hline $\mathrm{Al}$ & -0.003 & 0.000 & 0.000 \\
$\mathrm{Fe} 1$ & -0.013 & 0.013 & -0.001 \\
$\mathrm{Fe} 2$ & 0.014 & 0.016 & 0.014 \\
$\mathrm{Fe} 3$ & 0.000 & 0.000 & -0.016 \\
\hline
\end{tabular}

(b)

\begin{tabular}{cccc}
\hline \hline Atom & $L_{x}$ & $L_{y}$ & $L_{z}$ \\
\hline $\mathrm{Al}$ & -0.002 & 0.000 & 0.000 \\
$\mathrm{Fe} 1$ & -0.012 & 0.012 & 0.000 \\
$\mathrm{Fe} 2$ & 0.014 & 0.016 & 0.014 \\
$\mathrm{Fe} 3$ & 0.000 & 0.000 & -0.016 \\
\hline
\end{tabular}

are shown in Table 1. It is clear that the induced magnetic moment at $\mathrm{Al}$ is negative, showing that the $\mathrm{Fe}-\mathrm{Al}$ couples antiferromagnetically. It is interesting that the effects of the SOC is not so significant. Although Al decreases the Fe1 magnetic moment, the magnetic moments of $\mathrm{Fe} 2$ and Fe3 are the same with the pure bcc Fe value $\left(\sim 2.23 \mu_{\mathrm{B}}\right)$ at the optimized lattice constant [4]. This suggests that the effects of the Al impurity are well screened within the nearest neighbor. Note that the similar behavior was also discovered in the bcc $\mathrm{Ni}_{1} \mathrm{Fe}_{26}$ system [18]. We thus conclude that the first nearest neighbor magnetic interactions are playing a vital role in the magnetism of bcc Fe, and depending on the impurity atoms, the magnetic moment of Fel can be increased or decreased [4, 6, 18, 19].

To further demonstrate the effects of SOC on the magnetism of $\mathrm{Al}_{1} \mathrm{Fe}_{26}$, the orbital magnetic moments of the $\mathrm{Al}, \mathrm{Fe} 1, \mathrm{Fe} 2$, and $\mathrm{Fe} 3$ atoms are shown in Table 2. For comparison purposes, the unrelaxed and relaxed values of the magnetic moments are shown. One can see that atomic relaxation has only an effect on the Fe1 atoms. In the unrelaxed case, the orbital magnetic moments of Fel are in the $\left[\begin{array}{ll}\overline{1} & 1 \\ 1\end{array}\right]$ direction, whereas in the relaxed case, these are directed in the $[\overline{1} 10]$ direction. It is of imperative note that the orbital magnetic moments direction of the Fe1 is similar to pure bcc Fe [19]. It is also important
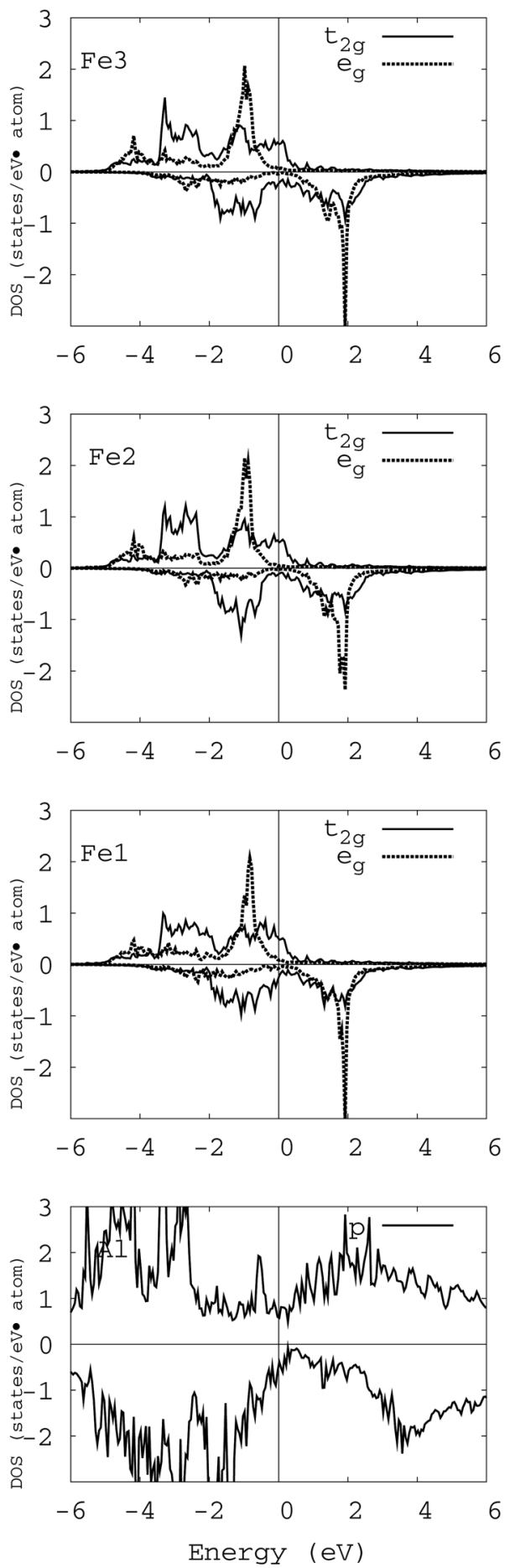

Fig. 2. Calculated orbital projected density of states (DOS) of $\mathrm{Al}, \mathrm{Fe} 1, \mathrm{Fe} 2$, and $\mathrm{Fe} 3$ atoms with spin-orbit coupling (SOC). The DOS values of the $p$ states are multiplied by a factor of 20. The upper and lower panel shows spin-up and spin-down states, respectively. The Fermi level $\left(E_{\mathrm{F}}\right)$ was set to zero. The solid and broken lines represent the $t_{2 g}$ and $e_{g}$ states of Fe.

to note [19] that the orbital magnetic moments of the Fe1 atoms in the bcc $\mathrm{Si}_{1} \mathrm{Fe}_{26}$ and $\mathrm{Al}_{1} \mathrm{Fe}_{26}$ are directed in the 
(110)

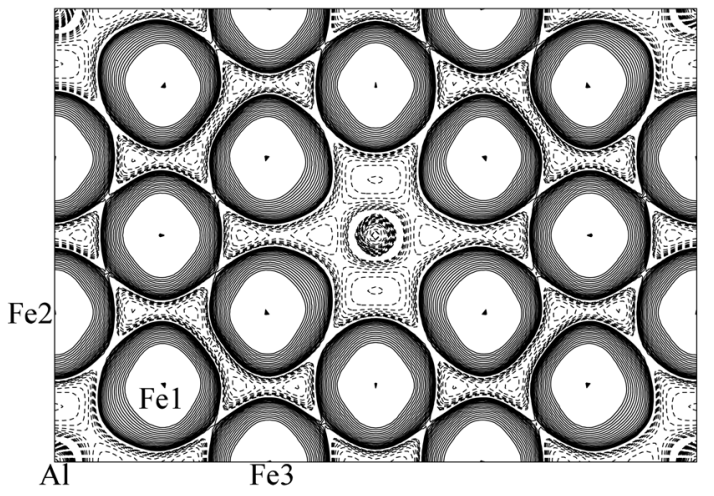

Fig. 3. Spin-density contour plot of relaxed $\mathrm{Al}_{1} \mathrm{Fe}_{26}$ calculated with SOC. The solid lines show positive spin density, whereas the broken lines show negative density. The contour starts from $5 \times 10^{4}$ electrons/a.u. ${ }^{3}$ and decrease by a factor $\sqrt{ } 2$.

same direction, [ $\overline{1} 10]$. Though the orbital magnetic moments are directed in the same direction in the $\mathrm{Al}_{1} \mathrm{Fe}_{26}$ and $\mathrm{Si}_{1} \mathrm{Fe}_{26}$, the magnitude of the orbital magnetic moment of $\mathrm{Fe} 1$ is different for signifying the different hybridization between the $\mathrm{Al}_{1} \mathrm{Fe}_{26}$ and $\mathrm{Si}_{1} \mathrm{Fe}_{26}$.

To see the Fe1-Al interactions thoroughly, we present the electronic density of states (DOS) of the $\mathrm{Al}_{1} \mathrm{Fe}_{26}$ in Fig. 2, where the solid and broken lines represent $t_{2 g}$ and $e_{g}$ states of the Fe atoms, respectively. The Fermi level $\left(E_{\mathrm{F}}\right)$ was set to zero. The $d$ states of the Fe are decomposed into $e_{g}$ and $t_{2 g}$ states. One can easily see strong hybridization between the $\mathrm{Fe} 1$ and $\mathrm{Al}$ atoms; this strong hybridization of $\mathrm{Fe} 1$ with $\mathrm{Al}$ causes reduction of the magnetic moment of Fe1 compared with $\mathrm{Fe} 2$ and Fe3. Near $E_{\mathrm{F}}$, the Fel $t_{2 \mathrm{~g}}$ and $\mathrm{Al} p$ bonding is dominated in the minority spins where one can see a small peak at Fe1, just below $E_{\mathrm{F}}$. The majority spin DOS of $t_{2 \mathrm{~g}}$ at $E_{\mathrm{F}}$ is flat for the $\mathrm{Fe} 2$ and $\mathrm{Fe} 3$ atoms. However, the electronic density of $t_{2 \mathrm{~g}}$ decays rapidly and delocalizes the $t_{2 \mathrm{~g}}$ electrons due to the covalent nature of $\mathrm{Fe} 1-t_{2 \mathrm{~g}}$ and $\mathrm{Al}-p$ orbitals at $E_{\mathrm{F}}$. The $\mathrm{Fe} 2$ and $\mathrm{Fe} 3$ atoms are far from the $\mathrm{Al}$ atoms and do not strongly interact with the $\mathrm{Al}$ atoms; the $\mathrm{DOS}$ of $\mathrm{Fe} 2$ and $\mathrm{Fe} 3$ are similar to pure bcc Fe [4]. Further determination of the spin density contours would give an idea about the nature of magnetic coupling between the magnetic elements. The spin density contour corrected with SOC in the (110) plane of the $\mathrm{Al}_{1} \mathrm{Fe}_{26}$ is shown in Fig. 3. This figure shows that the induced magnetic moment at $\mathrm{Al}$ is negative, represented by dashed lines. The Fel and $\mathrm{Al}$ atoms couple antiferromagnetically with each other, consistent with the local magnetic moments within the MT spheres. The antiferromagnetic interactions of $\mathrm{Fe} 1$ with Al decrease the local magnetic moment of the Fe1, com- pared with $\mathrm{Fe} 2$ and $\mathrm{Fe} 3$. The $\mathrm{Fe} 2$ and $\mathrm{Fe} 3$ atoms also interact antiferromagnetically with $\mathrm{Al}$, but this interaction is not sufficiently strong to decrease the local magnetic moment due to short-range nature of the antiferromagnetic coupling.

\section{Summary}

Using ab-initio calculations, the magnetic and electronic properties of $3.7 \mathrm{at} \% \mathrm{Al}$ in bcc $\mathrm{Fe}$ were studied. We considered the effects of spin-orbit (SOC) coupling on the magnetism and electronic structures of $\mathrm{Al}_{1} \mathrm{Fe}_{26}$. The calculations herein showed that SOC did not change the magnetic moments at the $\mathrm{Al}$ and $\mathrm{Fe}$ sites. The electronic density of states showed a strong hybridization between the $\mathrm{Fe} 1$ and $\mathrm{Al}$ atoms; this strong hybridization decreased the local magnetic moments of Fe1. The spin density contours revealed that the $\mathrm{Fe} 1$ and $\mathrm{Al}$ atoms interact antiferromagnetically and decrease the magnetic moment of the Fe1 compared with Fe2 and Fe3.

\section{Acknowledgements}

This work was supported by the POSCO Steel Innovation Program and by the Basic Science Research Program (Grant No. 20090088216) by the National Research Foundation by the Ministry of Education, Science, and Technology of Korea.

\section{References}

[1] V. K. Sikka, D. Wilkening J. Liebetrau, and B. Mackey, Mater. Sci. Eng. A 258, 229 (1998).

[2] B. V. Reddy and S. C. Deeve, Intermetallics 8, 1369 (2000).

[3] J. Bystrzycki, Intermetallics 8, 89 (2000).

[4] G. Rahman, I. G. Kim, and H. K. D. H. Bhadeshia, Phys. Rev. B 81, 184423 (2010).

[5] G. Rahman, I. G. Kim, and S. K. Chang, J. Korean Magn. Soc. (in Korean) 18, 211 (2008).

[6] C. T. Liu, E. H. Lee, and C. G. McKamey, Scripta Metall. 23, 875 (1989).

[7] J. R. Cullen, A. E. Clark, M. Wun-Fogle, J. B. Restorff, and T. A. Lograsso, J. Magn. Magn. Mater. 226, 948 (2001).

[8] Y. Ushigami, M. Mizokami, M. Fujikura, T. Kubota, H. Fujii, and K. Murakami, J. Magn. Magn. Mater. 254, 307 (2003).

[9] E. Wimmer, H. Krakauer, M. Weinert, and A. J. Freeman, Phys. Rev. B 24, 864 (1981), and references therein; M. Weinert, E. Wimmer, and A. J. Freeman, ibid. 26, 4571 (1982).

[10] See http://www.flapw.com 
[11] J. P. Perdew, K. Burke, and M. Ernzerhof, Phys. Rev. Lett. 77, 3856 (1996); ibid. 78, 1396(E) (1997).

[12] J.-H. Lee, T. Shishidou, and A. J. Freeman, Phys. Rev. B 66, 233102 (2002).

[13] D. D. Koelling and B. N. Harmon, J. Phys. C 10, 3107 (1977).

[14] R. Wu, D. Wang, and A. J. Freeman, J. Magn. Magn. Mater. 132, 103 (1994).

[15] M. Weinert, G. Schneider, R. Podloucky, and J. Redinger,
J. Phys.: Condens. Matter 21, 084201 (2009).

[16] S.-W. Seo, Y. Y. Song, G. Rahman, I. G. Kim, M. Weinert, and A. J. Freeman, J. Magnetics 14, 137 (2009).

[17] F. Birch, Phys. Rev. 71, 809 (1947); F. D. Murnaghan, Proc. Natl. Acad. Sci. U.S.A. 30, 244 (1944).

[18] G. Rahman and I. G. Kim, J. Magnetics 13, 124 (2008).

[19] J. Y. Lee, G. Rahman, I. G. Kim, and H. K. D. H. Bhadeshia, to be published elsewhere. 